\title{
BMJ Open Effects of chronic ethnic discrimination in the daily life of Turkish immigrants living in Austria: study protocol of a 30- day ambulatory assessment study
}

\author{
Andreas Goreis (D) , ${ }^{1,2}$ Urs M Nater, ${ }^{1}$ Ricarda Mewes ${ }^{2}$
}

To cite: Goreis A, Nater UM, Mewes R. Effects of chronic ethnic discrimination in the daily life of Turkish immigrants living in Austria: study protocol of a 30-day ambulatory assessment study. BMJ Open 2021;11:e046697. doi:10.1136/ bmjopen-2020-046697

- Prepublication history and additional supplemental materia for this paper are available online. To view these files, please visit the journal online (http://dx.doi.org/10.1136/ bmjopen-2020-046697)

Received 06 November 2020 Accepted 22 September 2021

Check for updates

(C) Author(s) (or their employer(s)) 2021. Re-use permitted under CC BY-NC. No commercial re-use. See rights and permissions. Published by BMJ.

${ }^{1}$ Department of Clinical and Health Psychology, Faculty of Psychology, University of Vienna, Vienna, Austria

${ }^{2}$ Outpatient Unit for Research, Teaching and Practice, Faculty of Psychology, University of Vienna, Vienna, Austria

\section{Correspondence to} Ricarda Mewes; ricarda.nater-mewes@univie. ac.at

\section{ABSTRACT}

Introduction Chronic ethnic discrimination is associated with negative mental and physical health outcomes in ethnic minority groups. It is assumed that suffering from repeated discriminatory events leads, over time, to psychological consequences such as higher perceived stress, higher negative affect and lower positive affect. Higher stress reactivity to non-discriminatory stressors, such as daily hassles, as well as anticipation and avoidance behaviour regarding discriminatory events, may further contribute to the overall burden for affected individuals. Studies investigating chronic ethnic discrimination and its psychological consequences in the daily lives of affected persons are lacking. Here, we present a study protocol to investigate the impact of chronic ethnic discrimination and acute discriminatory events in the daily lives of Turkish immigrants living in Austria, using an ambulatory assessment design. The feasibility of our study design was tested and confirmed in a pilot study $(n=10)$.

Methods and analysis Ninety male Turkish immigrants will complete daily questionnaires for 30 days. Participants will indicate stress, perceived discrimination, negative and positive affect, daily hassles, anticipation and avoidance behaviour, as well as rumination with regard to discriminatory events on a daily basis. Furthermore, they will use preprogrammed iPods to assess acute discriminatory events in real time. Our hypotheses will be tested using multilevel analyses.

Ethics and dissemination This study has been approved by the institutional review board of the University of Vienna (reference number 00358). Results will be presented at conferences and submitted for publication in a peerreviewed journal.

\section{INTRODUCTION}

Numerous studies have documented the negative impact of ethnic discrimination on mental and physical health. ${ }^{1-4}$ If frequent experiences of ethnic discrimination are encountered in day-to-day life, they may be perceived as threatening and require the mobilisation of internal resources. ${ }^{5}$ Chronic exposure to discriminatory events may thus constitute a persistent social stressor in
Strengths and limitations of this study

- This is the first study to investigate the direct and time-lagged effects of chronic ethnic discrimination on psychological consequences in the daily life of Turkish immigrants.

- Using an ambulatory assessment design, exposure to discriminatory events will be assessed by electronic devices in the daily life of Turkish immigrants in real-time and over 30 days.

- Psychological consequences (ie, perceived stress, affect, stress reactivity to daily hassles, anticipation and avoidance behaviour, as well as rumination) will be assessed using a daily diary design.

- Two groups will be compared: Turkish immigrants who experience chronic ethnic discrimination and Turkish immigrants who rarely experience ethnic discrimination in their lives

- This study uses self-report measures, and potential effects due to assessment reactivity (ie, changes in participant experiences and behaviour) may arise.

ethnic minority groups, potentially leading to psychological consequences such as negative affect, ${ }^{5}$ higher stress reactivity to nondiscriminatory stressors ${ }^{6} 7$ and maladaptive coping behaviours. ${ }^{8-10}$ However, the mechanisms through which chronic ethnic discrimination (CED) leads to impaired health are not fully understood. A stress and coping framework ${ }^{11-13}$ suggests the investigation of psychological mechanisms as well as potential protective factors, coping strategies and resources that may help persons to deal with the psychological consequences of ethnic discrimination. ${ }^{114}$

Most studies in this area of research are cross-sectional in nature and do not examine individuals on a day-to-day level. The framework of ambulatory assessment methodswhich includes daily diaries and experience sampling ${ }^{15}$-allows for reports of discriminatory events and relevant outcomes over longer periods of time and in real-time, rendering 


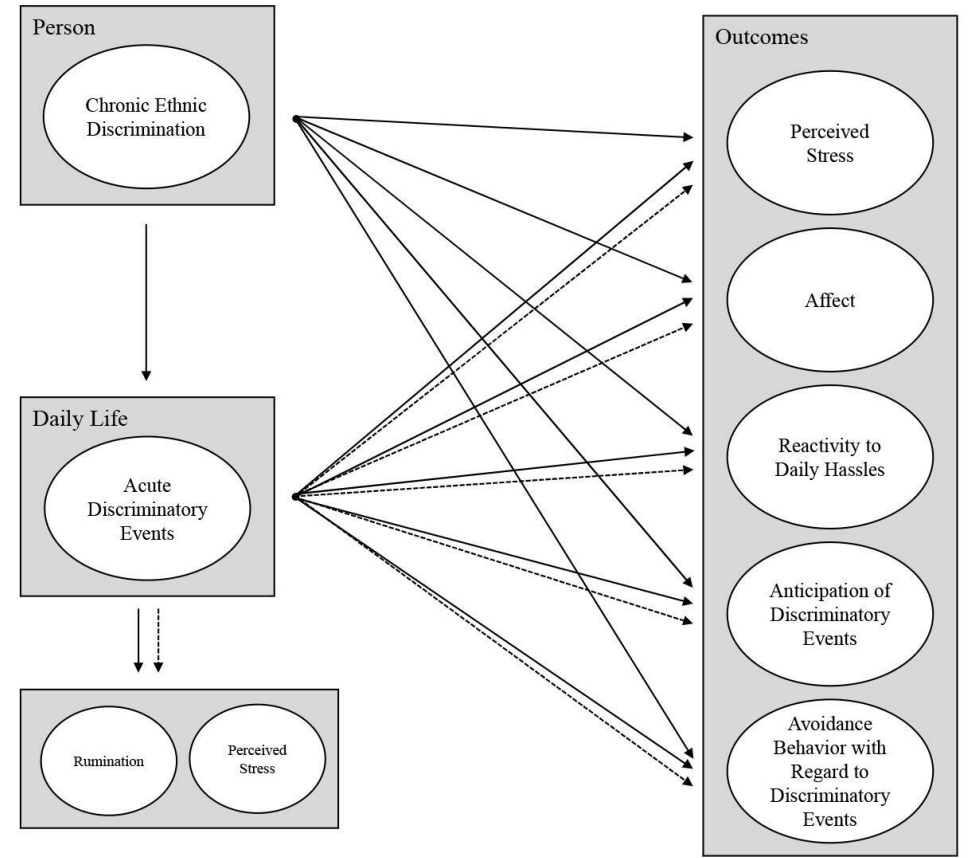

Figure 1 Model of the effects of chronic ethnic discrimination and acute discriminatory events that we plan to investigate in the proposed study.

it possible to investigate between-person and withinperson effects without recall bias. ${ }^{16} 17$ Compared with single assessments, an ambulatory assessment approach provides highly reliable, ecologically valid and repeated assessments of the outcomes of interest. ${ }^{18} 19$ Therefore, we present a study protocol of an ambulatory assessment study that aims to investigate the effects of both CED and acute discriminatory events on stress, affect, stress reactivity to daily hassles, anticipation of discriminatory events and avoidance behaviour with regard to discriminatory events. We plan to investigate these factors over 30 days in the daily lives of Turkish immigrants living in Austria, taking into account direct and indirect (ie, timelagged) variables. See figure 1 for an overview of the study variables.

\section{Chronic ethnic discrimination as a stressor}

Depending on individual factors, any event could be perceived as stressful. ${ }^{12}$ Two fundamental mechanisms determine the psychological stress reaction-the exposure and the reactivity to stressful events. ${ }^{20}$ In individuals who experience CED, discriminatory events occur frequently and the effects of these events have to be endured over an extended period of time (similar to non-discriminatory chronic stressors). ${ }^{21}{ }^{22}$ It has been suggested that the frequent exposure to chronic stressors, such as discriminatory events, depletes the capability to cope with the stress elicited by these potentially threatening and harmful events. ${ }^{52}$ The combination of the frequent stress exposure and the burdensome use of coping resources may thus, over time, lead to a higher reactivity to discriminatory events as they continue to occur in daily life. ${ }^{54}$
The effects of acute ethnic discrimination in daily life, that is, the psychological responses following a discriminatory event, have not been investigated in detail. Few studies have explored responses to acute discriminatory events in a standardised way in the laboratory. ${ }^{25}{ }^{26}$ However, they were limited to single discriminatory events ${ }^{27}$ or exclusion paradigms $^{28}$ and did not investigate responses to acute ethnic discrimination in everyday life. Acute discriminatory events in everyday life may occur in many forms and across contexts, that is, they may be subtle or overt, intentional or unintentional, interpersonal or online. All those events may elicit acute psychological responses if they are perceived as discriminatory by the person.

Most studies in the literature have focused on the impact of overt forms of discrimination (eg, threatening behaviour, name-calling), whereas subtle and covert ethnic discrimination has not been investigated as frequently. ${ }^{29}$ Subtle ethnic discrimination encompasses behaviours that are low in intensity, and which can easily be denied by perpetrators. ${ }^{30}$ This distinct form of ethnic discrimination has also been investigated under the label ethnic/ racial microaggressions, ${ }^{31}{ }^{32}$ and it seems to have similar detrimental effects on individuals to overt forms. ${ }^{29}{ }^{33} 34$ It is, therefore, important to account for the different forms of ethnic discrimination when investigating the daily life of ethnic minority groups. In our ambulatory assessment study, any discriminatory event, irrespective of its form, will be reported and included in our analyses.

\section{Affect}

Evidence suggests an association between a higher frequency of ethnic discrimination and more negative affect $^{535}$ as well as less positive affect, ${ }^{36}$ supporting the 
notion that well-being may be substantially reduced by the emotional impact of discriminatory events. ${ }^{37} 38$ Negative affect is a further risk factor for impaired mental and physical health. ${ }^{39}$ In addition to cross-sectional studies, ${ }^{40}{ }^{41}$ daily diary studies have also found ethnic discrimination to be an indicator of low levels of daily negative affect. ${ }^{73442-44}$

\section{Stress spillover to daily hassles}

The impact that arises from repeated exposure to discriminatory events may also contribute to an accumulation of perceived stress in other domains. ${ }^{7546}$ According to this model of stress spillover, the frequent experience of stressful discriminatory events may amplify the reactivity to daily hassles, that is, to negative everyday life events that are potentially stressful. ${ }^{47}$ Studies have found that people who are chronically exposed to ethnic discrimination are more likely to report a greater number of stressful life events. ${ }^{48}$ As different sources of stress in daily life may interact, it is necessary to capture the frequency of and reactivity to daily hassles in a differentiated way in order to understand how CED leads to deleterious psychological outcomes in minority groups.

\section{Coping strategies}

Coping refers to various cognitive or behavioural efforts that one undertakes in order to manage situations that are appraised to exceed, strain or tax personal resources. ${ }^{113}$ The anticipation of future occurrences of discriminatory events might be a predictive factor for the adverse health impact of CED. ${ }^{50}$ Several researchers have emphasised that anticipatory and heightened vigilance may constitute a coping strategy that leads to intrusive thoughts or images and may play an important role in determining the negative effects of stressors on health. ${ }^{46}$ Other maladaptive coping strategies in this context are avoidance behaviour and rumination. Avoidance behaviour may occur after discriminatory events have happened and may manifest in behaviours such as not thinking about the discriminatory event and reducing social contact due to fear of renewed discrimination. ${ }^{52}$ Moreover, avoidance behaviour seems to be a predictor of general life stress in Mexican Americans ${ }^{953}$ and has been associated with worse mental health status. ${ }^{54-56}$ Rumination is the tendency to passively perseverate on negative feelings and problems, ${ }^{57}$ and it has been found to increase symptoms of depression ${ }^{58}$ and negative affect. ${ }^{59}$ Potentially, ruminating on negative experiences (eg, after a discriminatory event) exacerbates and prolongs the already existing emotions, further leading to heightened stress. $^{60}$

\section{PROPOSED STUDY}

Taken together, past research has illustrated the damaging effects of ethnic discrimination on health. ${ }^{461}$ Suffering from CED both leads to higher stress and has a negative impact on affect, and also spills over to other domains.
Further proximal predictors, such as anticipation and avoidance behaviour, as well as protective factors like coping strategies, have been suggested to play a key role in the processes that ultimately lead to negative effects of ethnic discrimination. Past studies-even longitudinal and diary studies-had limitations in this regard, as they merely looked at single predictors alone, had a limited sampling time period (from 24 hours up to 14 days; with only a small number of studies sampling for 20 days $^{62}{ }^{63}$ ), or did not distinguish between the effects of CED and acute discriminatory events. . $^{5-16}{ }^{36} 64-66$ Due to these limitations of previous studies in this field, we plan to conduct the outlined study in order to add to the understanding of processes that ultimately lead to negative health outcomes for minority groups.

In our proposed study, we will focus on male Turkish immigrants living in Austria. Persons with a Turkish migration background constitute the largest group of non-EU citizens in Austria and other European countries. ${ }^{67}$ They are often the target of discrimination, ${ }^{68}$ and populationbased studies clearly indicate that Turkish immigrants are at increased risk of mental disorders and stress-related physical illnesses. ${ }^{69} 70$ Only males will be included since this study is part of a series of studies that also investigates biological factors of CED (eg, using hair cortisol and salivary cortisol levels as outcomes). Due to a greater variability in cortisol levels in females depending on the menstrual cycle phase or the use of hormonal contraceptives, ${ }^{71}$ we chose to focus on male participants in these studies. Moreover, women may experience discrimination based on the combination of gender and ethnic background (ie, intersectional discrimination ${ }^{72}$ ). Since we aim to specifically focus on the consequences of ethnic discrimination, intersectional discrimination would be a potentially confounding factor that we wanted to reduce.

To thoroughly investigate the differential impact of CED on Turkish immigrants living in Austria, we will employ an ambulatory assessment method in a sample of individuals who experience CED and in a comparison group comprising Turkish immigrants who rarely experience ethnic discrimination in their lives (rare ethnic discrimination; RED). Our study will utilise daily diary assessments to depict the effects of CED and acute discriminatory events on perceived stress, affect, stress reactivity to daily hassles, anticipation and avoidance, for 30 consecutive days.

Our hypotheses are as follows:

1. Turkish immigrants who experience CED will report higher perceived stress, lower positive affect, higher negative affect, a higher stress reactivity to daily hassles, higher anticipation of discriminatory events and higher avoidance behaviour with regard to discriminatory events than Turkish immigrants who experience RED.

2. On days when acute discriminatory events occur, Turkish immigrants who experience CED will report higher perceived stress, lower positive affect, higher negative affect, a higher stress reactivity to daily hassles, high- 
er anticipation of discriminatory events and stronger rumination than Turkish immigrants who experience RED.

3. Immediately after acute discriminatory events occur, Turkish immigrants who experience CED will report higher perceived stress than Turkish immigrants who experience RED.

4. On the days after the occurrence of acute discriminatory events, Turkish immigrants who experience CED will report higher perceived stress, lower positive affect, higher negative affect and a higher stress reactivity to daily hassles than on days when no acute discriminatory events occurred on the previous day.

5. On days when discriminatory events occur, Turkish immigrants who experience CED will report higher anticipation of discriminatory events and higher avoidance behaviour with regard to discriminatory events than on days when no acute discriminatory events occur.

\section{METHOD}

We present the method of our study protocol for the in-depth investigation of the immediate and prolonged effects of both chronic discrimination and acute discriminatory events in the daily life of Turkish immigrants. Participants in this study will provide reports in two ways: at the end of each day via a daily diary questionnaire, and directly after a discriminatory event has occurred via a preprogrammed iPod. In a pilot study conducted from December 2019 to March 2020, we set out to test the feasibility and acceptability of the study design using a small-scale sample of $n=10$ male Turkish immigrants. Ninety-four percent of all daily assessments were completed by the participants throughout the 30-day assessment, and six discriminatory events were reported. A detailed description of the pilot study is provided in the online supplemental material (see online supplemental table 1).

\section{Participants}

We aim to recruit twice as many participants in the CED group than in the RED group to have a sample size that is efficiently powered to compare the two groups over time (hypotheses 1-3) and to detect event-based effects in the CED group alone (hypotheses 4 and 5). We, therefore, conducted power analyses with the software powerlmm ${ }^{73}$ using the parameters of our pilot study (94\% completed assessments, a mean intraclass correlation of 0.39 and a mean within-variance ratio of 2.12; see online supplemental material. Simulations showed that with group sizes of $n=60$ and $n=30$, our study has a power of $82 \%$ to detect cross-level interactions ${ }^{74}$ with a medium effect size at $\alpha=0.05$. Therefore, we aim to include a total of $\mathrm{n}=90$ male participants $(\mathrm{n}=60$ Turkish immigrants who experience CED and n=30 Turkish immigrants who experience RED) $)^{75}$ in this study.

To be considered an immigrant, participants themselves, or at least one of their parents, have to have been born in Turkey (ie, first-generation or secondgeneration). Further inclusion criteria are male sex, age between 18 and 65 years, sufficient command of the German language, no alcohol or drug abuse, no medical illnesses, no mental disorders and a body mass index between 18 and $30 \mathrm{~kg} / \mathrm{m}^{2}$.

\section{Procedure}

For our study, we will recruit participants via advertisements in public places (eg, local shops), and on social media platforms. The study will be conducted in the laboratories of the Faculty of Psychology in Vienna, Austria. Persons who express an interest in participating will undergo a telephone interview and will be screened regarding our eligibility criteria. The absence of a mental disorder will be determined via individual items from the German version of the Structured Interview for Diagnostic and Statistical Manual of Mental Disorders, fourth edition, Axis I Disorders ${ }^{76}$ and the Patient Health Questionnaire (PHQ) ${ }^{77}$ Furthermore, as our main focus is on the investigation of Turkish immigrants who experience CED, we will ask participants to complete a 10-item version of the Everyday Discrimination Scale (EDS) ${ }^{78}$ in a German translation ${ }^{79}$ via telephone. The EDS is one of the most widely used measures of perceived discrimination and captures aspects of interpersonal discrimination in daily life. ${ }^{50}$ Participants are instructed to report how often they are treated unfairly because of their Turkish background. Items include daily experiences such as being treated with less respect, being treated with less courtesy and people acting as if they think that one is not smart, and will be rated on a 6-point Likert-type scale (1never, 6 -almost every day). The EDS scores will be coded according to a novel approach proposed by Michaels $e t$ $a l,{ }^{75}$ which provides a more nuanced exposure assessment than traditional frequency scores, thereby allowing for the comparison of two extreme value groups (ie, CED and RED). In this approach, responses are adjusted to the number of days per year, resulting in the following anchors: 1 (never) $=0 ; 2$ (less than once a year) $=0.5 ; 3$ (a few times a year) $=3 ; 4$ (a few times a month $)=36 ; 5$ (at least once a week $)=104 ; 6$ (almost every day $)=260$. A summed value can be calculated-with a potential range from 0 to 2600-referring to the total number of discriminatory events per year (ie, the chronicity). In their study, Michaels et $a l^{75}$ provided evidence for the robustness and sensitivity of this scale-coding approach, and proposed cutoffs with scores of $<25$ indicating rare exposure to ethnic discrimination and scores of $>481$ indicating chronic exposure. For the proposed study, we will use a slightly adapted cut-off score for CED $(>500)$ and retain $<25$ as a cut-off for RED. Potential participants will only be included in the planned study if they fall above or below these cut-off values.

After the screening procedure, participants will be invited to the laboratories of the Faculty of Psychology at the University of Vienna for an introductory session. During this introductory session, participants will 
provide written informed consent, complete the baseline measures and will be trained to handle the daily diary questionnaire and a preprogrammed iPod touch (iDia$\operatorname{logPad}, \mathrm{G}$ Mutz, Cologne, Germany) to report discriminatory events. The definition of discriminatory events (ie, any event that is perceived as discriminatory based on the persons' ethnic background) will be explained and examples of different forms of discriminatory events (eg, subtle, overt) will be provided and discussed. Participants will be instructed to report discriminatory events throughout the assessment time of 30 days via the iPod touch immediately after they are experienced. Additionally, they should provide answers to the daily diary questionnaire every day. The daily diary questionnaire will be completed via an internet survey platform (Unipark EFS Survey, Globalpark, Cologne, Germany), and emails providing a link to each day's questionnaire will be sent every evening at $8 \mathrm{p} . \mathrm{m}$. The daily diary questionnaire can be completed on a personal computer, smartphone or the provided iPod. To match repeated assessments on the daily diary questionnaire, all participants will be provided with an individual code, which they have to enter at every study assessment. Furthermore, a manual will be provided with detailed step-by-step descriptions of the procedure, handling of the iPod, responding to the different items and contact information of the study team.

After completing the study, participants will again be invited to our laboratories to return the iPods and for a postparticipation interview. Each participant will receive $100 €$ as compensation.

\section{Measures}

\section{Baseline}

The baseline measures will be presented to participants during the introductory session. First, participants will provide information on their age, sex and education. In the following, we will report the primary variables that are relevant for our hypotheses (ie, the daily diary variables). We will also assess secondary variables that are relevant in the field of ethnic discrimination and health but do not pertain to our specific hypotheses. These variables are cross-sectional and assess aspects of chronic stress, depressive and somatoform symptoms, potential protective factors (eg, in-group identification, acculturation, social support and emotion regulation), as well as personality traits. The secondary variables are listed and described in the online supplemental material, and an overview of all study variables and measures is provided in online supplemental table 2 .

\section{Daily diary}

In the daily diary assessment, participants will first be asked whether discriminatory events have occurred over the course of the day and if so, to provide detailed descriptions of the event in a text box. Subsequentlyand irrespective of the occurrence of acute discriminatory events-current perceived stress and perceived discrimination will be assessed with two items (ie, 'How stressed/ discriminated do you feel right now?'). Both items are rated on a 5-point Likert-type scale ranging from 1-not at all to 5-very much.

\section{Affect}

To assess daily positive and negative affect, the 20-item Positive and Negative Affect Schedule (PANAS) ${ }^{80}$ will be used. The PANAS is one of the most widely used scales to assess affect and was previously validated to assess daily affect. ${ }^{81}$ Positive affect (eg, excited, proud) and negative affect (eg, upset, afraid) will be assessed with 10 items each, with responses ranging from $1-$ not at all to 5-very much.

\section{Daily hassles}

Stress reactivity to daily hassles will be assessed with 18 items of the Daily Hassles Scales revised. ${ }^{82}$ The items cover negative events from six different domains of life: financial problems, time pressure, work hassles, environmental hassles, family hassles and health hassles. Daily hassles may occur without being perceived as stressful. Therefore, all items are rated on a 5-point Likert scale, with the response options 0 -did not occur, 1occurred, not stressful, 2-occurred, somewhat stressful, 3-occurred, moderately stressful and 4-occurred, very stressful.

\section{Rumination}

If a discriminatory event has occurred on a particular day, three items measuring rumination following discriminatory events will be presented. These items (' $I$ kept thinking about it', 'I re-enacted the situation in my mind' and 'I thought about the reasons why I was treated badly') will be rated on a 5-point Likert-type scale ranging from $1-$ not at all to 5 -very much. The items are adapted from the Anger Rumination Scale, ${ }^{83}$ which was previously used in the context of ethnic discrimination. $^{84}$

\section{Anticipation of discriminatory events}

The anticipation of discriminatory events will be assessed with three items (eg, 'I try to prepare for possible discriminatory events that may happen tomorrow') from the Racism-related Vigilance Scale. ${ }^{85}{ }^{86}$ Items will be rated on a 5-point Likert-type scale ranging from 1-not at all to 5-very much.

\section{Avoidance behaviour with regard to discriminatory events}

We will present four items assessing avoidance behaviour with regard to discriminatory events. These items (eg, 'Today, I avoided people or situations because I feared being discriminated against') are adapted from the avoidance subscale of the Coping Strategy Indicator, ${ }^{52}$ which was previously used to assess avoidance coping with regard to perceived ethnic discrimination in a sample of African-American students. ${ }^{55}$ Items will be rated on a 5-point Likert-type scale ranging from 1-not at all to 5very much. 


\section{Discriminatory events}

After experiencing acute discriminatory events during the day, participants should start an assessment by activating the iPod. Subsequently, they will be asked: 'Did you encounter a discriminatory event?', followed by: 'What exactly has happened?', with a range of response options: 'threatened', 'called names or insulted', 'attacked', 'treated as if I knew little/taken for a fool', 'offered advice and opinions I didn't want', 'treated worse, received poorer service', 'denied entry (eg, night club)' and 'other'. These response options were derived from several resources in the literature on unfair treatment and ethnic discrimination ${ }^{78788}$ and aim to account for different forms of discriminatory events (ie, overt and subtle, as well as everyday discrimination).

Subsequently, participants will be asked how many persons (from a list of 1-10 or more), and who, discriminated against them, with the response options 'familiar person(s)', 'unfamiliar person(s)', 'the police', 'medical personnel', 'vendor/salesperson', 'service personnel', 'doorman' and 'other'. It is possible to choose multiple options (eg, to choose both familiar person(s) and service personnel to report a waiter who is familiar to the participant) in order to facilitate nuanced responses. The next question asks about the specific area of life in which the event happened, with the response options 'work', 'spare time', 'shopping', 'restaurant/eating or drinking out', 'dealing with government agencies', 'doctor's visit/healthcare setting', 'internet/online gaming' and 'other'. These responses were adapted from a representative study on discrimination by the German Federal Anti-Discrimination Agency. ${ }^{89}$ Finally, two items will be presented assessing perceived stress and perceived discrimination during the discriminatory event (ie, 'How stressed did you feel in this situation' and 'How discriminated did you feel in the situation?'), both rated on a 5-point Likert type scale, ranging from 1-not at all to 5very much.

\section{Data analysis}

Analysis will be conducted using the software IBM SPSS $25,{ }^{90}$ HLM $7.03^{91}$ and R 3.6. ${ }^{92}$ Descriptive statistics of baseline variables and discriminatory events will be presented, and our hypotheses will be analysed using multilevel models. Repeated data entries at level 1 (ie, stress, affect, reactivity to daily hassles, anticipation of discriminatory events, avoidance behaviour with regard to discriminatory events and rumination) will be nested in participants (level 2).

For hypothesis 1 (effects of CED in daily life), we will compute models with the time-invariant predictor group coded as $0 / 1$ (RED/CED) as a random intercept and the daily diary variables as outcomes.

For hypothesis 2 (effects of acute discriminatory events in daily life), random slope models will be computed. These models include the time-invariant predictor group as a random intercept, the time-varying predictor event coded as $0 / 1$ (acute discriminatory event occurred: no/yes, as indicated in the daily diary assessments) as a random slope, and the cross-level interaction group $\times$ event. The outcomes will be the daily diary variables. These models will allow us to explain the variance in the effect of the level 1 predictor event with a level 2 predictor group. $^{93}$

For hypothesis 3 (immediate effects of acute discriminatory events in daily life), we will compute one model with the time-invariant predictor group as a random intercept and the outcome perceived stress immediately after acute discriminatory events occurred. Analyses pertaining to this hypothesis are only possible if the structure of the data permits-especially regarding the frequency and distribution of discriminatory events in the rare discrimination group. Therefore, we may adjust the analyses of hypothesis 3 after data collection is complete (ie, compare immediate stress of the chronic group with a proxy of daily stress in the rare group).

For hypothesis 4 (prolonged effects of acute discriminatory events), random slope models will be computed. These models will include the predictor group as a random intercept and a time-varying predictor event -1 coded as 0/1 (acute discriminatory event occurred on the day before: no/yes, as indicated in the daily diary assessments) denoting the day after the discriminatory event as a random slope. The outcomes will be the daily diary variables. If discriminatory events occurred on two (or more) consecutive days, only the day following the most recent discriminatory event will be included in the analyses.

Finally, for hypothesis 5 (anticipation of discriminatory events and avoidance behaviour with regard to discriminatory events), two models will be computed. These models will include the time-invariant predictor group as a random intercept, the time-varying predictor event coded as $0 / 1$ (acute discriminatory event occurred: no/yes, as indicated in the daily diary assessments) as a random slope, and the cross-level interaction group $\times$ event. The outcomes will be the anticipation and avoidance behaviour variables, respectively, from the daily diary questionnaire.

\section{Ethics and dissemination}

The study protocol and the corresponding pilot study were approved by the institutional review board of the University of Vienna (reference number 00358). Informed consent will be obtained by all participants in writing during the introductory session (see Procedure). Data will be collected and stored in a pseudonymised manner with a coded ID stored separately in locked cabinets. Only the project team has access to these cabinets and any personalised data or identifiers. After data collection has finished, all personalised data and identifiers will be deleted. The results of this study will be presented at conferences and submitted to a peer-reviewed journal.

\section{Patient and public involvement}

There was no involvement of participants or the public in the development of this study protocol. The results of 
this study will be forwarded to interested participants and disseminated via a peer-reviewed journal and at scientific conferences. Furthermore, the results will be disseminated and communicated to relevant stakeholders, persons working in (mental) healthcare and/or persons affected by ethnic discrimination.

\section{ANTICIPATED RESULTS/DISCUSSION}

Guided by stress and coping frameworks as well as models of ethnic discrimination, the proposed study aims to examine the direct and indirect effects that both CED and acute discriminatory events exert on stress and stressrelated outcomes in Turkish immigrants living in Austria. As noted in our hypotheses, we expect that experiencing CED will lead to reports of more negative outcomes and, furthermore, that acute discriminatory events will have additional negative effects on our outcomes.

The study is based on a pilot study that we conducted to evaluate the feasibility and acceptability of our design. In the pilot study, we were able to recruit a sufficient number of participants in a reasonable period of time, and the compliance with the daily diary assessments was very high. We, therefore, conclude that the design of our pilot study is feasible and can be implemented on a larger scale. However, the cross-sectional reports of perceived ethnic discrimination (ie, the classification of CED according to the EDS, using the cut-off by ${ }^{9495}$ were discrepant with the occurrence during the sampling period, as only six acute discriminatory events were reported by six persons. This was unexpected, as the number of discriminatory events did not fully correspond to the subpopulation of immigrants we intended to investigate (ie, Turkish immigrants suffering from CED). We conclude that a higher cut-off value should be chosen for inclusion as a chronically discriminated (CED) person in the proposed ambulatory assessment study. Therefore, we will classify participants based on chronicity scores suggested by Michaels $e t a l^{75}$ (see Procedure in the Study Protocol).

Several biases may potentially influence the results of our study. Only males will participate in our study and this focus on one gender will not allow us to generalise our findings to other genders. Thus, we may capture discriminatory events that are more often experienced by males than by females and are not able to assess events exclusively experienced by females. However, one large meta-analysis ${ }^{4}$ found that gender did not influence the associations between ethnic discrimination and mental or physical health. Selection bias may occur, that is, the participants of our study may not be fully representative of the population of Turkish immigrants living in Austria, since only healthy, non-obese participants that score below or above the cutoffs in the EDS are included. These inclusion criteria are necessary since the goal of our study is to investigate discrimination based on ethnicity as precisely as possible and we are thus unable to assess the impact of ethnic discrimination on unhealthy or impaired participants. In addition, selection bias may arise by our inclusion criteria of a sufficient command of the
German language as persons who are experiencing discrimination based on their language skills are not assessed. Furthermore, and due to the language inclusion criteria, our sample may represent a group of persons who are more integrated into Austrian society. Internet access at home will be required for participation, however, internet penetration rates are relatively high in Austria with $89 \%$ of all persons in Austria having internet access ${ }^{96}$ and internet access is often provided for free at restaurants, cafés, or public organisations.

We assume that the inclusion criteria regarding language and internet access are of little relevance since the sample we recruited for our pilot study provided the first evidence that our recruitment strategy and the design are feasible for people with a broad range of socioeconomic backgrounds. We attempt to limit biases regarding social desirability or assessment reactivity (due to the long assessment period) with a thorough briefing and training during the introductory session and by providing a study manual to participants. Importantly, the results of our study will be interpreted with caution and in light of all putative biases.

Overall, investigating the direct and indirect ways in which ethnic discrimination may impact the everyday life of ethnic minority groups is crucial to facilitate the understanding of the link between ethnic discrimination and health. Moreover, our findings will inform the development of ecological momentary interventions that target the negative effects of ethnic discrimination in the daily lives of affected persons.

\section{Twitter Andreas Goreis @andigoreis}

Contributors $A G, U M N$ and RM contributed to the conception and design of the study protocol. AG collected the data for the pilot study, analysed the data and wrote the first version of the manuscript. RM was the principal investigator of the study and was a major contributor in writing the manuscript. UMN provided critical revisions to the manuscript. All authors approved the final version of the manuscript and provided approval for publication.

Funding The authors acknowledge funding from the University Research Platform 'The Stress of Life: Processes and Mechanisms underlying Everyday Life Stress' (no grant number). The authors also acknowledge open access funding provided by the University of Vienna.

Competing interests None declared.

Patient consent for publication Consent obtained directly from patient(s)

Provenance and peer review Not commissioned; externally peer reviewed.

Supplemental material This content has been supplied by the author(s). It has not been vetted by BMJ Publishing Group Limited (BMJ) and may not have been peer-reviewed. Any opinions or recommendations discussed are solely those of the author(s) and are not endorsed by BMJ. BMJ disclaims all liability and responsibility arising from any reliance placed on the content. Where the content includes any translated material, BMJ does not warrant the accuracy and reliability of the translations (including but not limited to local regulations, clinical guidelines, terminology, drug names and drug dosages), and is not responsible for any error and/or omissions arising from translation and adaptation or otherwise.

Open access This is an open access article distributed in accordance with the Creative Commons Attribution Non Commercial (CC BY-NC 4.0) license, which permits others to distribute, remix, adapt, build upon this work non-commercially, and license their derivative works on different terms, provided the original work is properly cited, appropriate credit is given, any changes made indicated, and the use is non-commercial. See: http://creativecommons.org/licenses/by-nc/4.0/.

ORCID iD

Andreas Goreis http://orcid.org/0000-0001-8994-964X 


\section{REFERENCES}

1 Brondolo E, Brady Ver Halen N, Pencille M, et al. Coping with racism: a selective review of the literature and a theoretical and methodological critique. J Behav Med 2009;32:64-88.

2 Williams DR, Lawrence JA, Davis BA, et al. Understanding how discrimination can affect health. Health Serv Res 2019;54 Suppl 2:1374-88.

3 Brondolo E, Love EE, Pencille M, et al. Racism and hypertension: a review of the empirical evidence and implications for clinical practice. Am J Hypertens 2011;24:518-29.

4 Pascoe EA, Smart Richman L. Perceived discrimination and health: a meta-analytic review. Psychol Bull 2009;135:531-54.

5 Brondolo E, Brady N, Thompson S, et al. Perceived racism and negative affect: analyses of trait and state measures of affect in a community sample. J Soc Clin Psychol 2008;27:150-73.

6 Burrow AL, Ong AD. Racial identity as a moderator of daily exposure and reactivity to racial discrimination. Self and ldentity 2010;9:383-402.

7 Ong AD, Fuller-Rowell T, Burrow AL. Racial discrimination and the stress process. J Pers Soc Psychol 2009;96:1259-71.

8 Gerrard M, Stock ML, Roberts ME, et al. Coping with racial discrimination: the role of substance use. Psychol Addict Behav 2012;26:550-60.

9 Farley T, Galves A, Dickinson LM, et al. Stress, coping, and health: a comparison of Mexican immigrants, Mexican-Americans, and nonhispanic whites. J Immigr Health 2005;7:213-20.

10 Alvarez AN, Juang LP. Filipino Americans and racism: a multiple mediation model of coping. J Couns Psychol 2010;57:167-78.

11 Harrell SP. A multidimensional conceptualization of racism-related stress: implications for the well-being of people of color. Am J Orthopsychiatry 2000;70:42-57.

12 Clark R, Anderson NB, Clark VR, et al. Racism as a stressor for African Americans. A biopsychosocial model. Am Psychol 1999;54:805-16.

13 Lazarus RS, Folkman S. Stress, appraisal, and coping. New York, NY: Springer, 1984

14 Brondolo E, Thompson S, Brady N, et al. The relationship of racism to appraisals and coping in a community sample. Ethn Dis 2005;15:S5-14-19.

15 Schneider S, Stone AA. Ambulatory and diary methods can facilitate the measurement of patient-reported outcomes. Qual Life Res 2016;25:497-506.

16 Fuller-Rowell TE, Nichols OI, Burrow AL, et al. Day-to-day fluctuations in experiences of discrimination: associations with sleep and the Moderating role of internalized racism among African American college students. Cultur Divers Ethnic Minor Psychol 2021;27:107-17

17 Ohly S, Sonnentag S, Niessen C, et al. Diary studies in organizational research: an introduction and some practical recommendations. $J$ Pers Psychol 2010;9:79-93.

18 Stone AA, Shiffman S. Ecological momentary assessment (EMA) in behavioral medicine. Ann Behav Med 1994;16:199-202.

19 Ebner-Priemer UW, Trull TJ. Ambulatory assessment: an innovative and promising approach for clinical psychology. Eur Psychol 2009;14:109-19.

20 Bolger N, Zuckerman A. A framework for studying personality in the stress process. J Pers Soc Psychol 1995;69:890-902.

21 Turner RJ, Wheaton B, Lloyd DA. The epidemiology of social stress. Am Sociol Rev 1995:60:104.

22 Turner HA, Turner RJ. Understanding variations in exposure to social stress. Health 2005;9:209-40.

23 Gallo LC, Matthews KA. Understanding the association between socioeconomic status and physical health: do negative emotions play a role? Psychol Bull 2003;129:10-51.

24 Rozanski A, Blumenthal JA, Kaplan J. Impact of psychological factors on the pathogenesis of cardiovascular disease and implications for therapy. Circulation 1999;99:2192-217.

25 Busse D, Yim IS, Campos B, et al. Discrimination and the HPA axis: current evidence and future directions. J Behav Med 2017;40:539-52.

26 Korous KM, Causadias JM, Casper DM. Racial discrimination and cortisol output: a meta-analysis. Soc Sci Med 2017;193:90-100.

27 Fischer S, Nater UM, Strahler J, et al. Psychobiological impact of ethnic discrimination in Turkish immigrants living in Germany. Stress 2017;20:167-74.

28 Peterson LM, Stock ML, Monroe J, et al. Racial exclusion causes acute cortisol release among emerging-adult African Americans: the role of reduced perceived control. J Soc Psychol 2020;160:658-74.

29 Jones KP, Peddie Cl, Gilrane VL, et al. Not so subtle: a meta-analytic investigation of the correlates of subtle and overt discrimination. $J$ Manage 2016;42:1588-613.
30 Cortina LM. Unseen injustice: incivility as modern discrimination in organizations. Acad Manage Rev 2008;33:55-75

31 Sue DW, Capodilupo CM, Torino GC, et al. Racial microaggressions in everyday life: implications for clinical practice. Am Psychol 2007;62:271-86.

32 Sue DW, Bucceri J, Lin Al, et al. Racial microaggressions and the Asian American experience. Cultur Divers Ethnic Minor Psychol 2007;13:72-81.

33 Noh S, Kaspar V, Wickrama KAS. Overt and subtle racial discrimination and mental health: preliminary findings for Korean immigrants. Am J Public Health 2007;97:1269-74.

34 Ong AD, Burrow AL, Fuller-Rowell TE, et al. Racial microaggressions and daily well-being among Asian Americans. J Couns Psychol 2013;60:188-99.

35 Landrine $\mathrm{H}$, Klonoff EA. The schedule of racist events: a measure of racial discrimination and a study of its negative physical and mental health consequences. J Black Psychol 1996;22:144-68.

36 Smart Richman L, Pek J, Pascoe E, et al. The effects of perceived discrimination on ambulatory blood pressure and affective responses to interpersonal stress modeled over 24 hours. Health Psychol 2010;29:403-11.

37 Yoo HC, Lee RM. Ethnic identity and approach-type coping as moderators of the racial discrimination/well-being relation in Asian Americans. J Couns Psychol 2005;52:497-506.

38 Schmitt MT, Branscombe NR, Postmes T, et al. The consequences of perceived discrimination for psychological well-being: a metaanalytic review. Psychol Bull 2014;140:921-48.

39 Suls J, Bunde J. Anger, anxiety, and depression as risk factors for cardiovascular disease: the problems and implications of overlapping affective dispositions. Psychol Bull 2005;131:260-300.

40 Noh S, Kaspar V. Perceived discrimination and depression: Moderating effects of coping, acculturation, and ethnic support. Am $J$ Public Health 2003;93:232-8.

41 Klonoff EA, Landrine H, Ullman JB. Racial discrimination and psychiatric symptoms among blacks. Cult Divers Ethn Minor Psychol 1999:5:329-39.

42 Broudy R, Brondolo E, Coakley V, et al. Perceived ethnic discrimination in relation to daily moods and negative social interactions. J Behav Med 2007;30:31-43.

43 Taylor TR, Kamarck TW, Shiffman S. Validation of the Detroit area study discrimination scale in a community sample of older African American adults: the Pittsburgh healthy heart project. Int J Behav Med 2004;11:88-94.

44 Swim JK, Hyers LL, Cohen LL, et al. African American college students' experiences with everyday racism: characteristics of and responses to these incidents. J Black Psychol 2003;29:38-67.

45 Pearlin LI, Aneshensel CS, LeBlanc AJ. The forms and mechanisms of stress proliferation: the case of AIDS caregivers. J Health Soc Behav 1997;38:223-36.

46 Pearlin LI, Schieman S, Fazio EM, et al. Stress, health, and the life course: some conceptual perspectives. J Health Soc Behav 2005;46:205-19.

47 Kanner AD, Coyne JC, Schaefer C, et al. Comparison of two modes of stress measurement: daily hassles and uplifts versus major life events. J Behav Med 1981;4:1-39.

48 Pieterse AL, Carter RT. An examination of the relationship between general life stress, racism-related stress, and psychological health among black men. J Couns Psychol 2007;54:101-9.

49 Cichy KE, Stawski RS, Almeida DM. Racial differences in exposure and reactivity to daily family stressors. J Marriage Fam 2012;74:572-86.

50 Williams DR, Mohammed SA. Discrimination and racial disparities in health: evidence and needed research. J Behav Med 2009;32:20-47.

51 Baum A, Cohen L, Hall M. Control and intrusive memories as possible determinants of chronic stress. Psychosom Med 1993;55:274-86.

52 Amirkhan JH. A Factor Analytically Derived Measure of Coping - the Coping Strategy Indicator. J Pers Soc Psychol 1990;59:1066-74.

53 Villegas-Gold R, Yoo HC. Coping with discrimination among Mexican American college students. J Couns Psychol 2014;61:404-13

54 Aranda MP, Castaneda I, Lee PJ, et al. Stress, social support, and coping as predictors of depressive symptoms: gender differences among Mexican Americans. Soc Work Res 2001;25:37-48.

55 Barnes PW, Lightsey OR. Perceived Racist discrimination, coping, stress, and life satisfaction. J Multicult Couns Devel 2005;33:48-61.

56 Utsey SO, Ponterotto JG, Reynolds AL, et al. Racial discrimination, coping, life satisfaction, and self-esteem among African Americans. $J$ Couns Dev 2000;78:72-80.

57 Nolen-Hoeksema S, Wisco BE, Lyubomirsky S. Rethinking Rumination. Perspect Psychol Sci 2008;3:400-24. 
58 Nolen-Hoeksema S, Morrow J. Effects of rumination and distraction on naturally occurring depressed mood. Cogn Emot 1993;7:561-70.

59 Brosschot JF, Gerin W, Thayer JF. The perseverative cognition hypothesis: a review of worry, prolonged stress-related physiological activation, and health. J Psychosom Res 2006;60:113-24.

60 Robinson MS, Alloy LB. Negative cognitive styles and stress-reactive rumination interact to predict depression: a prospective study. Cognit Ther Res 2003;27:275-91.

61 Paradies Y. A systematic review of empirical research on selfreported racism and health. Int J Epidemiol 2006;35:888-901.

62 Hoggard LS, Byrd CM, Sellers RM. The lagged effects of racial discrimination on depressive symptomology and interactions with racial identity. J Couns Psychol 2015;62:216-25.

63 Hoggard LS, Byrd CM, Sellers RM. Comparison of African American college students' coping with racially and nonracially stressful events. Cult Divers Ethn Minor Psychol 2012;18:329-39.

64 Torres L, Ong AD. A daily diary investigation of Latino ethnic identity, discrimination, and depression. Cultur Divers Ethnic Minor Psychol 2010;16:561-8.

65 Zeiders K. Discrimination, daily stress, sleep, and mexican-origin adolescents' internalizing symptoms. Cultur Divers Ethn Minor Psychol 2017;23:570-5.

66 Hatzenbuehler ML, Nolen-Hoeksema S, Dovidio J. How does stigma "get under the skin"?: the mediating role of emotion regulation. Psychol Sci 2009;20:1282-9.

67 Statistics Austria. Population with foreign background, 2020. Available: http://www.statistik.at/web_de/statistiken/bevoelkerung/ bevoelkerungsstruktur/bevoelkerung_nach_migrationshintergrund/ index.html

68 Liebkind K, Jasinskaja-Lahti I, Jaakkola M, et al. The influence of experiences of discrimination on psychological stress: a comparison of seven immigrant groups. J Community Appl Soc Psychol 2000;10:1-16.

69 de Wit MAS, Tuinebreijer WC, Dekker J, et al. Depressive and anxiety disorders in different ethnic groups. Soc Psychiatry Psychiatr Epidemiol 2008;43:905-12.

70 Levecque K, Lodewyckx I, Vranken J. Depression and generalised anxiety in the general population in Belgium: a comparison between native and immigrant groups. J Affect Disord 2007;97:229-39.

71 Kirschbaum C, Kudielka BM, Gaab J, et al. Impact of gender, menstrual cycle phase, and oral contraceptives on the activity of the hypothalamus-pituitary-adrenal axis. Psychosom Med 1999;61:154-62.

72 Lewis TT, Cogburn CD, Williams DR. Self-Reported experiences of discrimination and health: scientific advances, ongoing controversies, and emerging issues. Annu Rev Clin Psychol 2015;11:407-40.

73 Magnusson K. Powerlmm: power analysis for longitudinal multilevel models, 2018. Available: https://github.com/rpsychologist/ powerlmm/

74 Maas CJM, Hox JJ. Sufficient sample sizes for multilevel modeling. Methodology 2005;1:86-92.

75 Michaels E, Thomas M, Reeves A, et al. Coding the everyday discrimination scale: implications for exposure assessment and associations with hypertension and depression among a cross section of mid-life African American women. J Epidemiol Community Health 2019;73:577-84

76 First MB, Spitzer RL, Gibbon M. Structured Clinical Interview for DSM-IV Axis I Disorders, Clinician Version (SCID-CV). Washington, D. C.: American Psychiatric Press, 1997.
77 Löwe B, Zipfel S, PHQ-D HW. Gesundheitsfragebogen für Patienten. Manual Komplettversion und Kurzform. 2. Auflage. Pfizer: Karlsruhe, 2002.

78 Williams DR, Yu Y, Jackson JS, et al. Racial differences in physical and mental health: socio-economic status, stress and discrimination. $J$ Health Psychol 1997;2:335-51.

79 Kunst JR, Sam DL, Ulleberg P. Perceived islamophobia: scale development and validation. Int. J. Intercult. Relations 2013;37:225-37.

80 Watson D, Clark LA, Tellegen A. Development and validation of brief measures of positive and negative affect: the PANAS scales. $J$ Pers Soc Psychol 1988:1063-70.

81 Rush J, Hofer SM. Differences in within- and between-person factor structure of positive and negative affect: analysis of two intensive measurement studies using multilevel structural equation modeling. Psychol Assess 2014;26:462-73.

82 Holm JE, Holroyd KA. The Daily Hassles Scale (Revised) - Does It Measure Stress or Symptoms. Behav Assess 1992;14:465-82.

83 Sukhodolsky DG, Golub A, Cromwell EN. Development and validation of the anger rumination scale. Pers Individ Dif 2001;31:689-700.

84 Borders A, Liang CTH. Rumination partially mediates the associations between perceived ethnic discrimination, emotional distress, and aggression. Cultur Divers Ethnic Minor Psychol 2011;17:125-33.

85 Clark R, Benkert RA, Flack JM. Large arterial elasticity varies as a function of gender and racism-related vigilance in black youth. $J$ Adolesc Health 2006;39:562-9.

86 Hicken MT, Lee H, Ailshire J, et al. "Every shut eye, ain't sleep": The role of racism-related vigilance in racial/ethnic disparities in sleep difficulty. Race Soc Probl 2013;5:100-12.

87 Mewes R, Asbrock F, Laskawi J. Perceived discrimination and impaired mental health in Turkish immigrants and their descendents in Germany. Compr Psychiatry 2015;62:42-50.

88 Sibley CG. The BIAS-Treatment scale (BIAS-TS): a measure of the subjective experience of active and passive harm and facilitation. $J$ Pers Assess 2011;93:300-15.

89 Beigang S, Fetz K, Kalkum D, et al. Diskriminierungserfahrungen in Deutschland, 2017. Available: http://www.antidiskriminierungs stelle.de/SharedDocs/Downloads/DE/publikationen/Expertisen/ Expertise_Diskriminierungserfahrungen_in_Deutschland.pdf?_blob= publicationFile\& $v=4$

90 IBM Corp. Ibm SPSS statistics for windows, version 25.0. Armonk, NY: IBM Corp, 2017.

91 Raudenbush SW, Bryk AS, Cheong YF, et al. Scientific software international. HLM 7. Lincolnwood, IL, 2011.

92 R Core Team. R: a language and environment for statistical computing. Vienna, Austria: R Foundation for Statistical Computing, 2019. http://www.r-project.org/

93 Aguinis H, Gottfredson RK, Culpepper SA. Best-Practice recommendations for estimating cross-Level interaction effects using multilevel modeling. J Manage 2013;39:1490-528.

94 Mays VM, Cochran SD. Mental health correlates of perceived discrimination among lesbian, gay, and bisexual adults in the United States. Am J Public Health 2001;91:1869-76.

95 Pérez DJ, Fortuna L, Alegría M. Prevalence and correlates of everyday discrimination among U.S. Latinos. J Community Psychol 2008;36:421-33.

96 Hootsuite \& We Are Social. Digital 2021 global digital overview. Austria, 2021. https://datareportal.com/reports/digital-2021-austria 